\title{
Application of a Volterra quadratic polynomial to modeling elements of heat engineering devices
}

\author{
Ekaterina Antipina ${ }^{1}$, Vadim Spiryaev ${ }^{1 *}$, Emir Tairov $^{1}$ \\ ${ }^{1}$ Melentiev Energy Systems Institute Siberian Branch of the Russian Academy of Sciences,Lermontov str.130,Irkutsk, \\ Russia
}

\begin{abstract}
This paper considers integral models built to describe dynamic processes in a $135 \mathrm{MW}$ power unit condenser. For this purpose, we use a quadratic segment of the Volterra integral power series. The first set of models was built with a perturbation of the cooling water flow, and the second one with a perturbation of the steam flow. For all sets of models, changes in pressure and temperature in the condenser, as well as temperature changes in LHP-1, were considered as a response to perturbation. For models built with perturbation of the cooling water flow velocity, we considered an extreme problem of finding optimal amplitudes of the input perturbations. The results of calculations proved to be sufficiently accurate.
\end{abstract}

\section{Introduction}

Much work is currently being done in Russia to develop and modernize generating facilities of thermal power plants, which prompts the need for an adequate description of how their components work. Thus, for example, the authors of [1] made analysis of the technology development for modeling the dynamic processes in thermal power plants, and in [2] the research object was the simplest concentrated heat exchanger.

Various methods of modeling heat and power equipment can be found in [3, 4]. In particular, [3] considers models of physical objects, such as block condensation steam turbine set, low-potential complex of combined heat and power plant and nuclear thermal power plants, feed water heater.

The authors of $[5,6,7,8]$ also modeled heat power equipment. In [5], they made calculations for the counter current heat exchanger for the input-output system of the heat carrier of the power unit with the VVER-440 reactor at the Kola NPP. In [6], the authors modeled burning processes in circulating fluidized bed boilers and in [7] they considered a steam-water heat exchanger installed in a circuit chemical water treatment plant. In [8], the problem of flow distribution in the energy plants with different pressure drop laws in the branches of complex gas air and steam-water ducts is considered.

In the above papers, studies are mainly based on the use of differential equations. At the same time, such mathematical tools as the Volterra series (polynomials) are being widely used and developed. For example, in [9] the Volterra polynomials are used to study the dynamics and perform the diagnostics of a vent-reluctance motor. The use of the Volterra polynomials in thermal power engineering was demonstrated in [10] for solving the problem of creating a method for the dynamic calculation of boiler units. And in [11,12], using the example of a heat exchanger model with independent heat supply, represented as an object with lumped parameters, the authors showed that an integral model based on a quadratic segment of the Volterra series can be employed to fairly accurately and quickly describe nonlinear transient processes in the heat exchanger. A detailed description of this object is presented in [13].

In this paper, the problem of modeling the dynamics of the condenser included in the power unit of Nazarovo power station using the Volterra polynomials is considered.

\section{Object of research}

The Nazarovo power station is one of the largest electricity producers in Eastern Siberia and the Russian Far East. It comprises seven power units, one with the power of $498 \mathrm{MW}$, and the other six are $135 \mathrm{MW}$ each.

The common scheme of a power unit of $135 \mathrm{MW}$ includes the following objects (Fig. 1): D is a combined deaerator, FEP is a system of feed electric pump, OTSG1, OTSG-2 is a once-through steam generator, $\mathrm{HPC}$ is a high-pressure casing, MPC is a medium pressure casing, LPC is a low pressure casing, BS is a boiler system, $\mathrm{C}$ is a condenser, CEP is a condensate electric pump system, the group of LPH is a low-pressure feed heater, the group of HPH is a high-pressure feed heater.

The simulation model of this unit was implemented in the form of "P150" software that simulates the power unit of the Irkutsk Central Heating and Power Plant (CHP-10) [14]. The mathematical model includes about 100

eldin@mail.ru 
algebraic-differential and 500 algebraic equations and closing relations.

The dotted line on Fig. 1 marks the section where we study nonlinear dynamics of pressure and temperature at the outlet of bulk heat exchangers, including the condenser type 80-KTC-1 and the LPH-1 from the group of LPH.

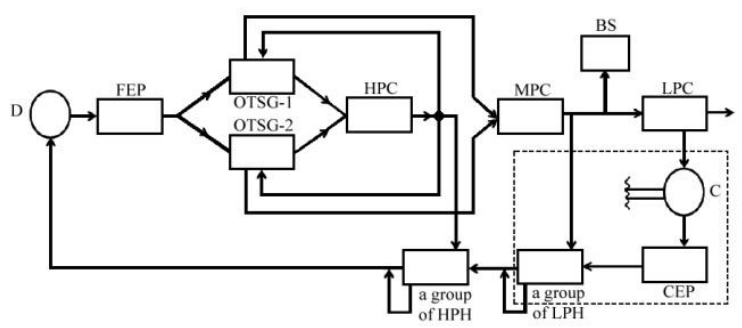

Fig.1. Block structural diagram of water-steam circuit of power unit.

Consider two cases. In the first one, it is assumed that the steam flow $D_{1 s}$ and $D_{2 s}$, coming from the LPC and MPC, respectively, to the condenser and LPH-1 are constant. The input signal for this model is the water flow $\Delta D_{w}$. Here $D_{1 s}=45.51 \mathrm{~kg} / \mathrm{s}$ and $D_{2 s}=2.03 \mathrm{~kg} / \mathrm{s}$. The second case implies that the steam flow $D_{2 s}$ and the water flow $D_{w}$ are constant. In this case, the input signal is the steam flow $\Delta D_{1 s}$. Here $D_{2 s}=2.03 \mathrm{~kg} / \mathrm{s}$ and $D_{w}=11562.2$ $\mathrm{kg} / \mathrm{s}$. The output signals in both cases are the pressure deviation $\Delta p$ and temperature deviation in the condenser $\Delta t_{1}$, the temperature deviation in LPH-1 $\Delta t_{2}$.

Figure 2 shows two cases of the section under consideration, where the blue signals are the input signals, and the green signals are the output signals.

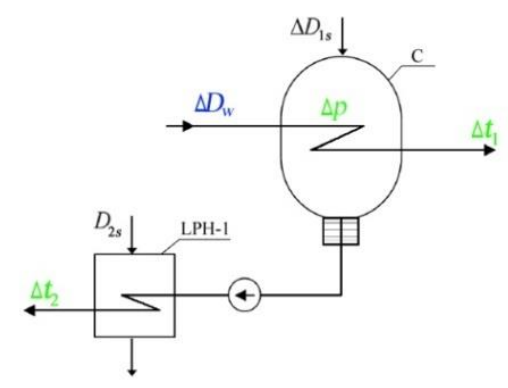

a)

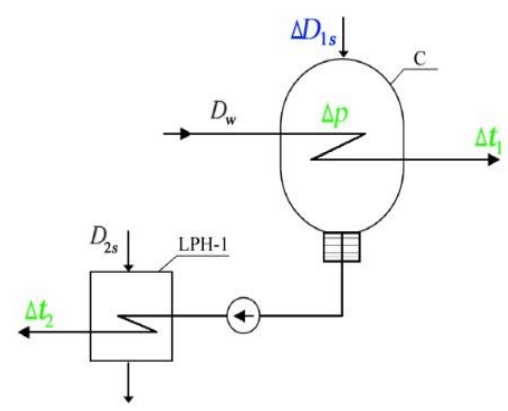

b)

Fig.2. Block diagram of the circuit area for the first (a) and second (b) cases.

Figure 3 shows block diagrams of three channels of the steam-water circuit of the power unit when the water flow (Fig. 3, a) or the steam flow (Fig. 3, b) is taken as an input signal, and the output signals for both cases are the change in pressure and the change in temperature in the condenser and LPH-1. Here, each channel will be called a mathematical model.

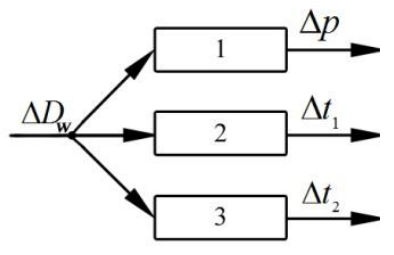

a)

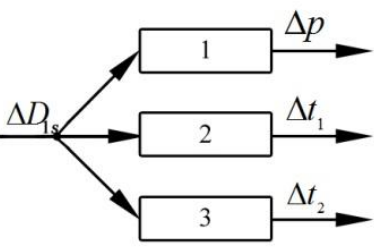

b)
Fig.3. Block diagram of three modeled sections of the steamwater circuit of a power unit. The case a), when the input signal is the water flow, and case b) when the input signal is the steam flow.

\section{Construction of mathematical models}

To build models of nonlinear dynamic systems, we apply the Volterra functional series, which represents a system's response to an external influence in the form of a finite segment (polynomial) of an integral power series

$$
y(t)=\sum_{n=1}^{N} \int_{0}^{t} \cdots \int_{0}^{t} K_{n}\left(t, s_{1}, \cdots s_{n}\right) \prod_{k=1}^{n} x\left(s_{k}\right) d s_{k}, \quad t \in[0, T]
$$

Where $y(t)$ and $x(t)$ are deviations of the input and output signals from their stationary values, $K_{n}, n=1,2, \ldots, N$ are the Volterra kernels to be identified. The parameter $N$ determines the number of terms in (1).

If the system is stationary, i.e., its dynamic characteristics remain the same throughout the transition process, the Volterra kernels do not depend on time. Then (1) can be rewritten as

$$
y(t)=\sum_{n=1}^{N} \int_{0}^{t} \cdots \int_{0}^{t} K_{n}\left(s_{1}, \cdots, s_{n}\right) \prod_{k=1}^{n} x\left(t-s_{k}\right) d s_{k}, \quad t \in[0, T] .
$$

Thus, the construction of a model in the form of a Volterra polynomial (2) means finding the required number of terms (parameter $N$ ) and identifying Volterra kernels. In this paper, we only assume that $N=2$. Identification will be based on the works $[10,15]$.

For all cases shown in Fig. 3 we write down the following quadratic Volterra polynomial:

$$
\begin{gathered}
y_{m}(\tau)=\int_{0}^{\tau} K_{1}^{(m)}\left(s_{1}\right) x\left(t-s_{1}\right) d s_{1}+ \\
+\int_{0}^{\tau} \int_{0}^{\tau} K_{2}^{(m)}\left(s_{1}, s_{2}\right) \prod_{l=1}^{2} x\left(t-s_{l}\right) d s_{l}, \tau \in[0, T],
\end{gathered}
$$

where the input signal $x(\tau)$ can play the role of the water flow $x(\tau)=\Delta D_{w}$ or the steam flow $x(\tau)=\Delta D_{1 s}$, and the output signal has three forms $y_{1}(\tau)=\Delta p, y_{2}(\tau)=\Delta t_{1}, y_{3}(\tau)=\Delta t_{2}$, at $m=1,2,3$. Building a model (3) means identifying its transient characteristics (Volterra kernels). For clarity, we will build models in three stages.

At the first stage, for the identification of polynomials of the form (3), two series of test (training) signals were 
carried out in order to receive responses $y_{m}(\tau)$ to the signals of the form

$$
x(\tau)=\alpha(\mathrm{e}(\tau)-\mathrm{e}(\tau-\omega)), \quad 0 \leq \omega \leq \tau \leq T,
$$

where $\mathrm{e}(\tau)$ is the Heaviside step function, $\alpha \neq 0$ is the amplitude of the test signals perturbation. To adjust the models, we selected the flow rate of water and steam with amplitudes $\pm 0.3 D_{0 w}$ and $\pm 0.1 D_{0 s}$, where $D_{0 w}=1156.2 \mathrm{~kg} / \mathrm{s}$ and $D_{0 s}=45.51 \mathrm{~kg} / \mathrm{s}$ are the initial values of water and steam flow, respectively. The time span was determined by the results of the evaluation testing of the studied dynamic systems. It was $T=120 \mathrm{~s}$.

All tests to obtain the required set of source data were carried out using the "P150" software [14].

At the second stage, taking into account the input perturbations (4) at $\alpha= \pm 0.3 D_{0 w}$ for the first case and at $\alpha= \pm 0.1 D_{\text {os }}$ for the second case, the problem of identification of Volterra kernels was solved by the Dinamika Software Package [16]. Thus, the six integral models were built using Volterra quadratic polynomial as a basis.

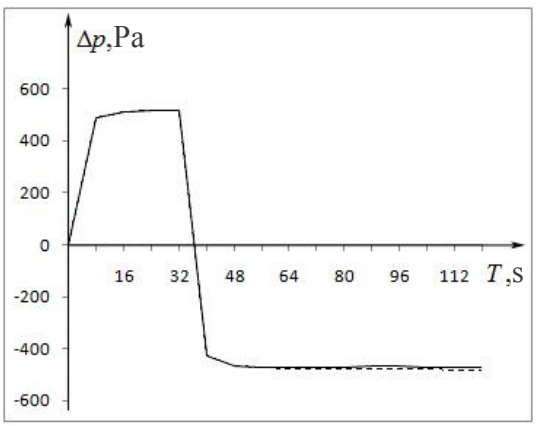

a)

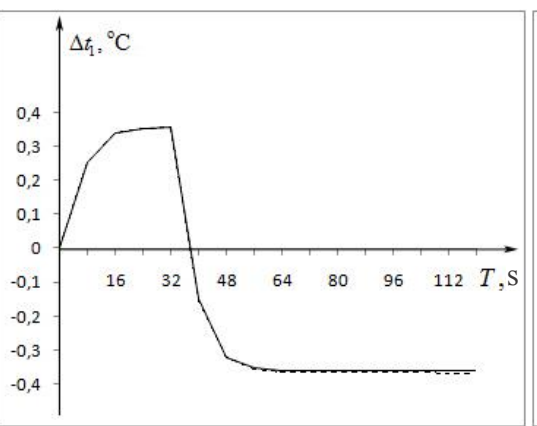

b)
For the implementation of the third stage (building models) another software was created in MATLAB. For each case, on the basis of the identified Volterra kernels, the integral models were constructed.

Testing of the integral models was carried out using the following signal:

$$
x(\tau)=\beta(\mathrm{e}(\tau)-2 \mathrm{e}(\tau-32))
$$

which differs from all test signals of the form (4).To test the model, in (5) we assume $\beta=0.35 D_{0 w}$ when the input signal is water flow, and $\beta=0.15 D_{0 s}$ when the input signal is steam flow. The signal (5) will be fed to the integral model and the reference model. By the reference model we understand the response of the "P150" software to the signal (5). In this case, we note that if we feed the same input signals that were used to identify the Volterra kernels $(\alpha=\beta)$, the error between the reference and the integral models will be zero.

Figure 4 and Fig. 5 show graphs of pressure and both temperatures for each input signal of the form (5) with $\beta=0.35 D_{0 w}$ for the water flow and $\beta=0.15 D_{0 s}$ for the steam flow.

Fig.4.Graphs of pressure a), the temperature in the condenser b) and the temperature in the LPH-1 c) with the input disturbance of steam flow. The solid line indicates the response of the reference model; the dotted line indicates the response of the integral model.

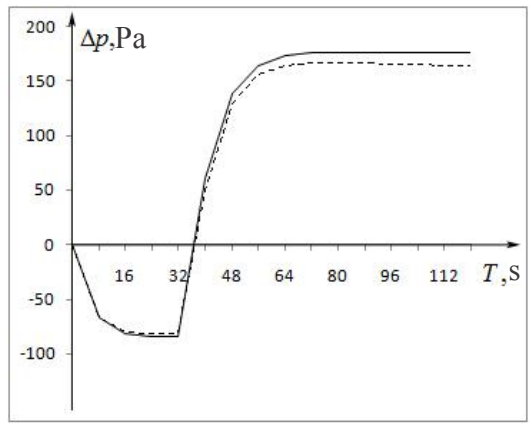

a)

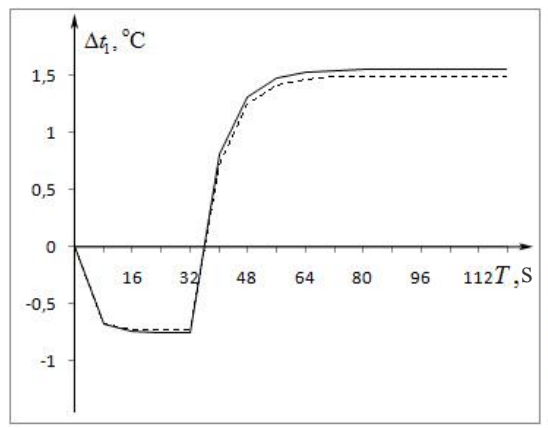

b)

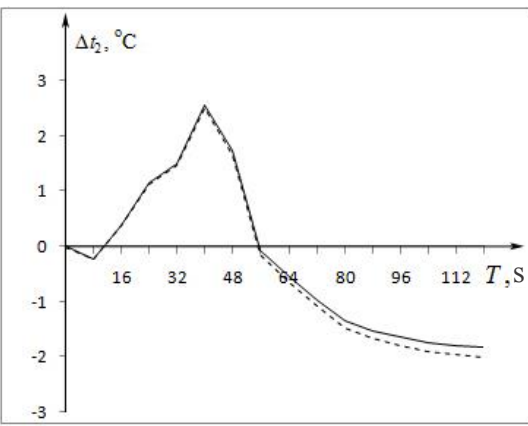

c)

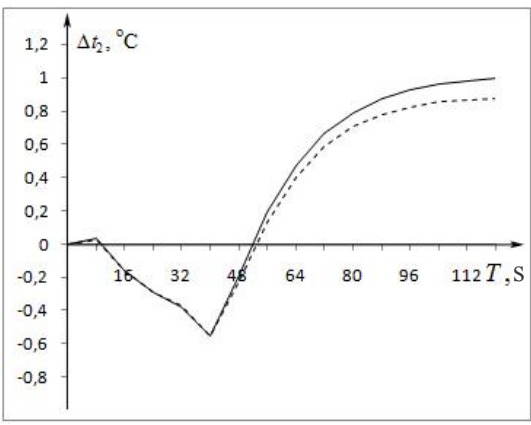

c)

Fig.5. Graphs of pressure a), the temperature in the condenser b) and the temperature in the LPH-1 c) with the input disturbance of water flow. The solid line indicates the response of the reference model; the dotted line indicates the response of the integral model.

In Fig. 4 and Fig. 5 we can see that the error in all three models has the same structure: at the beginning of the segment the error is close to zero, however, as it approaches the end of the segment, the error increases. The maximum error for the signal of the form (5) is usually achieved at the end of the segment. Therefore, we present just two tables to illustrate the accuracy of modeling.

Tables 1 and 2 presents the numerical interpretation of the error for steam flow (Fig. 4, b) and the numerical interpretation of the error for water consumption (Fig. 4, a), respectively.

Table 1. Error for steam flow perturbation.

\begin{tabular}{|c|c|c|}
\hline & $\varepsilon_{a(\max )}$ & $\varepsilon_{r(\max )}$ \\
\hline$\Delta p$ & $11,48 \mathrm{~Pa}$ & $2,5 \%$ \\
\hline$\Delta t_{1}$ & $0,0085^{\circ} \mathrm{C}$ & $2,4 \%$ \\
\hline$\Delta t_{2}$ & $0,1737^{\circ} \mathrm{C}$ & $9,5 \%$ \\
\hline
\end{tabular}


Table 2. Error for cooling water flow perturbation

\begin{tabular}{|c|c|c|}
\hline & $\varepsilon_{a(\max )}$ & $\varepsilon_{r(\max )}$ \\
\hline$\Delta p$ & $12,76 \mathrm{~Pa}$ & $7,2 \%$ \\
\hline$\Delta t_{1}$ & $0,085^{\circ} \mathrm{C}$ & $3,8 \%$ \\
\hline$\Delta t_{2}$ & $0,124^{\circ} \mathrm{C}$ & $12,4 \%$ \\
\hline
\end{tabular}

Here, the absolute error and ratio error are determined by the formulas:

$$
\begin{gathered}
\varepsilon_{a(\max )}=\max _{t_{i} \in[0,120]}\left|y_{e t}^{\beta}\left(t_{i}\right)-y_{\mathrm{int}}^{\alpha, \beta}\left(t_{i}\right)\right|, \\
\varepsilon_{r(\max )}=\max _{t,[0,120]}\left|\frac{y_{e t}^{\beta}\left(t_{i}\right)-y_{\mathrm{int}}^{\alpha, \beta}\left(t_{i}\right)}{y_{e t}^{\beta}\left(t_{i}\right)}\right| \cdot 100 \% .
\end{gathered}
$$

In (6) and (7) $y_{e t}^{\beta}$ is the response of the reference model to the signal (5) and $y_{\mathrm{int}}^{\alpha, \beta}$ is the response of the integral model to the signal (5).

To improve the accuracy of the models, the amplitudes of the input perturbations were optimized at the identification stage, for the case when the water flow is fed to the input. Similarly to [17], for input signals of the form

$$
x(\tau)=\beta \cdot \mathrm{e}(\tau), \beta \in[0, B] .
$$

we considered the following extreme problem:

$$
\alpha^{*}=\arg \min _{\alpha \in[0, B]} \max _{\beta \in[0, B]}|N(\alpha, \beta)|
$$

In (9) $\alpha$ is the amplitude of the quadratic model, $\beta$ is an arbitrary amplitude, $N(\alpha, \beta)=\max _{t,[0,120]}\left(y_{e t}^{\beta}\left(t_{i}\right)-y_{\mathrm{int}}^{\alpha, \beta}\left(t_{i}\right)\right)$, is the error between the reference and integral models for the perturbation (8). Our error calculation is totally different to that presented in [17], where the error is considered at the end of the modeling segment. In our case, it is necessary to calculate the maximum of the error on the entire segment of simulation and the maximum is not necessarily located at the end of the segment. Such error calculation allows us to obtain a specific type of error qualitatively similar to the one given in [17]. As a result, we will seek a solution to (9) by the brute force method. Graphic interpretation of this solution is presented in Fig. 6.

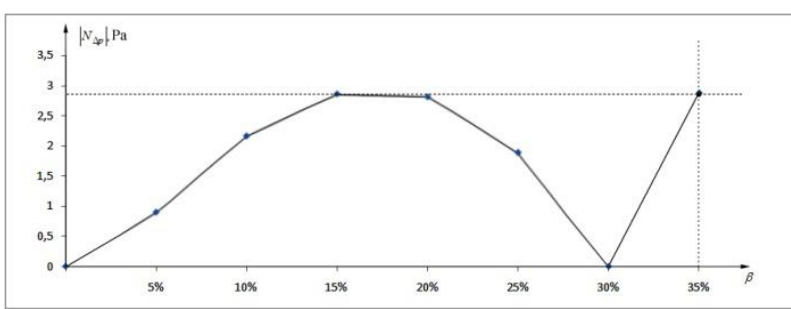

Fig.6. Graph of $N(\alpha, \beta)$ of the pressure dynamics for the first case.

Similarly, the optimal amplitudes were determined for the water temperatures in the condenser and LPH-1, respectively, for the first case. Estimates of these parameters are presented in Table 3.
Table 3. Optimal amplitudes for models of pressure dynamics and temperature dynamics in the condenser and LPH-1, respectively.

\begin{tabular}{|l|l|}
\hline$\alpha_{p}^{*}$ & $0,86 B$ \\
\hline$\alpha_{t_{i}}^{*}$ & $0,86 B$ \\
\hline$\alpha_{t_{2}}^{*}$ & $0,87 B$ \\
\hline
\end{tabular}

Table 3 shows that the values obtained in all three cases fall within the range of $0.75 \mathrm{~B} \div 0.9 \mathrm{~B}$, presented in [17].

Next, we determine the accuracy limits of the integral model depending on the perturbation. The following perturbations can be chosen as input signals:

$$
\begin{aligned}
& \Delta D_{w 1}=\beta \cdot \mathrm{e}(t), \\
& \Delta D_{w 2}=-\beta \cdot \mathrm{e}(t),
\end{aligned}
$$

where $\beta=[0,0.45]$. The admissible input signals were found with a ratio error determined by (7). The table for admissible values $\beta \leq \beta \leq \bar{\beta}$ with an error $\varepsilon_{r(\max )} \leq \varepsilon_{\max }$ for pressure in the condenser is given below.

Table 4. Admissible values of $\beta$ for models describing pressure in the condenser.

\begin{tabular}{|c|c|c|c|c|c|}
\hline \multirow{2}{*}{$\varepsilon_{\max } \%$} & \multicolumn{2}{|c|}{$\begin{array}{c}\text { Admissible values } \beta \\
\text { for }(10), \%\end{array}$} & & \multicolumn{2}{|c|}{$\begin{array}{c}\text { Admissible values } \\
\beta \text { for }(11), \%\end{array}$} \\
\cline { 2 - 3 } \cline { 5 - 5 } & $\underline{\beta}$ & $\bar{\beta}$ & $\underline{\beta}$ & $\bar{\beta}$ \\
\hline$[-10,-8)$ & 5 & 10 & 5 & 14 \\
\hline$[-8,-4)$ & 11 & 22 & & 15 & 22 \\
\hline$[-4,0]$ & 23 & 30 & 23 & 30 \\
\hline$[0,4]$ & 30 & 35 & 30 & 35 \\
\hline$(4,8]$ & 36 & 40 & & 36 & 40 \\
\hline$(8,10]$ & 41 & 44 & & 41 & 43 \\
\hline
\end{tabular}

As we can see, the greatest error in this case is achieved at the beginning and at the end of the segment $\beta=[0,0.45]$, and the smallest errors appear when approaching the values of amplitudes at which the Volterra kernels were identified. This trend is observed in models describing the temperature in the condenser and the LPH-1.

\section{Conclusions}

We considered two cases of the condenser operation. For each of them, we built mathematical models describing the effect of water flow and steam flow on the change in pressure, the temperature in the condenser, and temperature in the LPH-1. We obtained the admissible model performance using arbitrary signals (different to those for which the Volterra kernels were identified). For the case when the input signal is the water flow, we obtained the optimal amplitudes and the error ranges, which guarantee the specified accuracy.

The research was carried out under State Assignment, Project III.17.3.1 (reg.no.AAAA-A17-117030310442-8) and Project III.17.1.3 (reg.no.AAAA-A17117030310443-5) of the Fundamental Research of Siberian Branch of the Russian Academy of Sciences. 


\section{References}

1. A. Rubashkin, V. Rubashkin, Thermal Engineering, 51(10), 812-816 (2004)

2. A. Rubashkin, V. Verbitskii, V. Rubashkin, Thermal Engineering, 50(8), 659-663 (2003)

3. L. Popyrin, M.: Energiya, 416 (1978)

4. S. Novikov, Novosibirsk: NGTU, 64 (2004)

5. Chan Kuok Hung, E. Arakelyan, A. Ivanov, Teploenergetika, 6, 60-64 (1995)

6. A. Rychkov, Computational Technologies.- ICT SB RAS, 7, 94-105 (2002)

7. D. Telichenko, Information Science and Control System, 2(36), 153 -165 (2013)

8. A. Levin, V. Chistyakov, E.Tairov, Programming and Computer Software, 9(4), 53-62 (2016)

9. S. Grigorenko, V. Pavlenko, S. Pavlenko, A. Fomin, EEET, 4(11), 38-43 (2014)

10. K.Tibor, Teploenergetika, 12,71-75 (1969)

11. E. Tairov, A. Apartsyn, Applied Energy-New York$\mathrm{C} / \mathrm{C}$ Of Izvestiia - Rossiiskaia Akademiia Nauk Energetik,34, 60-71 (1996)

12. A. Apartsyn, E. Tairov, S. Solodusha, D. Khudyakov, Proc. of the Russian Academy of Sciences. Energy, 3, 2842 (1994)

13. E. Tairov, Bulletin of the Academy of Sciences of the USSR. Ser. Energy and Transport, 1, 150-156 (1989)

14. E. Tairov, A. Loginov, V. Chistyakov, Irkutsk:ESI SB RAS, Preprint № 11, 43(1999)

15. A. Apartsyn, VSP, Utrecht-Boston, 168 (2003)

16.S. Solodusha, Bulletin of the South Ural State University. Ser. Computer Technologies Automatic Control Radio Electronics, 17(2), 83-92 (2017)

17. A. Apartsyn, S. Solodusha, AURC, 65(3), 464-471 (2004) 